\title{
Neoadjuvant chemoradiotherapy followed by surgery in patients with unresectable locally advanced colon cancer: a prospective observational study
}

This article was published in the following Dove Press journal:

OncoTargets and Therapy

\author{
Hui Chang ${ }^{1,2, *}$ \\ $X$ in $\mathrm{Yu}^{1,2, *}$ \\ Wei-wei Xiao',2 \\ Qiao-xuan Wang ${ }^{1,2}$ \\ Wen-hao Zhou ${ }^{1,3}$ \\ Zhi-fan Zeng', \\ Pei-rong Ding ${ }^{1,3}$ \\ Li-ren $\mathrm{Li}^{1,3}$ \\ Yuan-hong Gao',2
}

'State Key Laboratory of Oncology in South China, Collaborative Innovation Center for Cancer Medicine, Guangzhou, China; ${ }^{2}$ Department of Radiation Oncology, Sun Yat-sen University Cancer Center, Guangzhou, China; ${ }^{3}$ Department of Colorectal Surgery, Sun Yat-sen University

Cancer Center, Guangzhou, China

*These authors contributed equally to this work

\begin{abstract}
Background: The prognosis of locally unresectable colon cancer (CC) is poor. This prospective observational study aimed to further evaluate the feasibility and efficacy of neoadjuvant chemoradiotherapy (NACRT) followed by surgery in these patients.

Patients and methods: We consecutively enrolled patients who were diagnosed with locally unresectable CC from November 2010 to March 2017, and received NACRT followed by surgery. The data of all the patients were collected prospectively. The R0 resection, downstage and pathologic complete response ( $\mathrm{pCR}$ ) rates were calculated to evaluate the short-term treatment effects. The overall survival (OS) was used to evaluate the long-term outcome. The incidence of NACRT-related acute toxicities and postsurgical complications were used to assess the safety.
\end{abstract}

Results: A total of 60 patients were eligible for analysis, including 57 (95.0\%) patients who attained resectability after NACRT. Among patients managed with surgery, 49 cases $(86.0 \%)$ achieved R0 resection, and 15 cases (26.3\%) achieved pCR. Down T stage was seen in 47 cases (82.5\%), and down $\mathrm{N}$ stage was seen in 53 cases (93.0\%). After a median follow-up time of 26 months, the OS appeared as $76.7 \%$. The most common grade 3/4 NACRT-related toxicity was myelosuppression (incidence, 20.0\%). The incidence of grade 3/4 surgery-related complication was $7.0 \%$.

Conclusion: NACRT might be a safe and effective choice for patients with locally unresectable $\mathrm{CC}$ to improve treatment effects, long-term survival and life quality, though further validation is needed.

Keywords: colon cancer, unresectable disease, R0 resection, complete response, survival

\section{Introduction}

Colorectal cancer is one of the most common malignancies throughout the world, including People's Republic of China. ${ }^{1} \mathrm{Up}$ to now, surgery remains the primary radical therapy for colon cancer (CC). ${ }^{2}$ Thus, resection radicality is one of the most important predictors for local recurrence and overall survival (OS). ${ }^{3}$ About $10 \%-15 \%$ of the CC patients are diagnosed with locally advanced disease. ${ }^{4}$ To attain a $\mathrm{R} 0$ resection, which is defined as a resection with microscopically negative margins, the en-bloc multivisceral resection (MVR) involving a partial or complete removal of the organs adhering to the primary tumor is performed in these patients. ${ }^{5}$ This extensive surgical procedure leads to complications with a total incidence rate as high as $20.3 \%{ }^{6}$ The postoperative complications might also influence the clinical outcome. ${ }^{7}$ Therefore, there is a need to
Correspondence: Li-ren Li;

Yuan-hong Gao

Department of Radiation Oncology, Sun Yat-sen University Cancer Center, 65I Dongfeng Road East, Guangzhou, Guangdong 510060, People's Republic of China

Tel +86 I38 2647 6766;

$+8613560182168$

Email lilr@sysucc.org.cn;

gaoyh@sysucc.org.cn 
improve resection radicality and reduce the surgery-related complications simultaneously, in order to ameliorate the prognosis of locally advanced CC. Moreover, $5 \%$ of the CC patients present with a locally unresectable disease, including tumors fixed to critical structures, and organs not amenable or appropriate for radical resection. ${ }^{8}$ Enhancing resectability could also improve the survival of patients with locally advanced CC, especially those with unresectable disease.

Like CC, locally advanced rectal cancer (RC) can also invade the adjacent structures. ${ }^{9}$ Neoadjuvant chemoradiotherapy (NACRT) has been proven to improve not only the resection radicality and survival but also the postsurgical life quality of locally advanced RC, especially in the era of intensity-modulated radiotherapy (IMRT). ${ }^{10,11}$ So, NACRT followed by surgery is now proposed by the National Comprehensive Cancer Network as the standard preoperative treatment for these patients. ${ }^{12}$ The most common $(57.0 \%)$ pathologic diagnosis of $\mathrm{RC}$ is the moderately differentiated adenomatous carcinoma, which also occupies the majority $(72.0 \%)$ of CC..$^{13,14}$ It is not hard to speculate that clinical outcome of patients with CC might also be ameliorated through NACRT. Actually, prior to this study, we had reported the effectiveness and safety of NACRT in 21 patients with unresectable sigmoid CC. ${ }^{15}$ Hence, this prospective observational study aimed to further validate the results of our previous work in an enlarged scale of unresectable CC patients.

\section{Patients and methods}

\section{Patient selection}

Patients with pathologically diagnosed and previously untreated CC in our hospital from November 1, 2010 to March 31, 2017 were initially considered. A patient would be consecutively enrolled into this study and prospectively observed if his/her tumor was considered unresectable and in need of NACRT, through the multidisciplinary team consultation. The reasons of unresectability mainly included the following: 1) preoperative imaging examinations showed that the tumor extensively invaded into the adjacent organs, such as the bladder, ureter, small intestine, pancreas or great vessels, to make the achievement of a clean radical margin impossible and 2) radical resection was deemed impossible after exploratory laparotomy. Patients with distant metastases were also eligible because the primary objective of this study was to assess the local response of the tumor. But the patients with the following criteria would be excluded: 1) age $<18$ or $>75$ years; 2) Karnofsky performance score $<70$; 3) severe hematopoietic, heart, lung, liver or kidney dysfunctions, which made the patients unsuitable for surgery or NACRT; and 4) prior history of other malignancies. This study was approved by the Institutional Review Board of Sun Yat-sen University Cancer Center. All the patients enrolled signed informed consent form before treatment.

\section{Diagnosis and staging work-up}

All the patients were pathologically diagnosed through a colonoscope. CC was defined as the primary tumor with a distance of at least $15 \mathrm{~cm}$ from its inferior margin to the anal verge under rigid proctoscopy. All the patients underwent a computed tomography (CT) scan of the chest and abdomen, a magnetic resonance imaging (MRI) of the pelvis, an endoscopic ultrasonography and a whole-body bone scan or positron emission tomography to evaluate their pretreatment clinical stage, which was determined according to the 7th edition of the Union for International Cancer Control/ American Joint Cancer Committee (UICC/AJCC) TNM staging system. ${ }^{16}$ The bladder involvement was confirmed by cystoscope. The pretreatment carcinoembryonic antigen (CEA) and carbohydrate antigen 19-9 (CA19-9) were also tested for each patient.

\section{Treatment strategies}

The technique of radiotherapy for all the patients in this study was IMRT, whose target definition, delineation and dose prescription were described in our previous work. ${ }^{15}$ For more details, refer to the Supplementary materials.

Chemotherapy concurrently with radiotherapy and after the surgery was performed with a XELOX (capecitabine + oxaliplatin) regimen every 21 days. Capecitabine (CAP) was given at a dose of $1,000 \mathrm{mg} / \mathrm{m}^{2}$ twice daily from Day 1-14. Oxaliplatin was given at a dose of $100 \mathrm{mg} / \mathrm{m}^{2}$ in concurrent chemotherapy, or $130 \mathrm{mg} / \mathrm{m}^{2}$ in adjuvant chemotherapy, on the first day of a chemotherapy cycle. When the age of the patients exceeded 70 years, a single-agent CAP was given instead of the XELOX regimen.

Pelvic MRI, thoracoabdominal CT, colonoscopy and all blood tests were repeated 4 weeks after NACRT. The surgery was scheduled 6-8 weeks after the radiotherapy. A colectomy with an en-bloc removal of the regional lymph nodes was performed. When tumor infiltrated or adhered to the adjacent organs, the surgeons would also apply an enbloc MVR, in which the attached organs were partially or entirely removed.

The postoperative pathology of the patients was assessed to decide the pathological stage, which was also based on UICC/AJCC TNM staging system. ${ }^{16}$ The indexes of shortterm treatment effects included the rate of $\mathrm{R} 0$ resection, 
downstage and pathologic complete response (pCR). pCR was defined as the absence of tumor cells in the primary site and regional lymph nodes of surgical samples. The acute toxicities of NACRT were evaluated based on the Common Terminology Criteria for Adverse Events (CTCAE) ver. 4.03. Postoperative complications were evaluated according to the Clavien-Dindo classification.

\section{Follow-up}

Outpatient follow-up was conducted every 3 months in the first 2 years after the surgery, and semiannually from the $3 \mathrm{rd}$ to 5 th year. Then the patients were followed up by outpatient interview or telephone, until death or July 31, 2017, whichever came first. The main contents of the follow-up included complete physical examination, thoracoabdominal CT scan, pelvic MRI scan, test of CEA and CA19-9 level, and annual colonoscopy and whole-body bone scan.

The indexes of the long-term outcome included the OS, the disease-free survival (DFS), the local-recurrence-free survival (RFS) and the distant-metastasis-free survival (MFS). The OS referred to the percentage of the patients who were alive after a certain time period from pathological diagnosis. The latter 3 indexes were defined as the percentage of patients who had no corresponding events after a certain time period from diagnosis. The events for DFS included death, local recurrence and distant metastasis. The events for RFS and MFS were local recurrence and distant metastasis, respectively.

\section{Statistical analysis}

Continuous data were presented as median with range, and categorical data were presented as proportions (\%). The OS, the DFS, the RFS and the MFS were calculated using the Kaplan-Meier approach. The survival curves were also depicted. Because the T stage was a key prognosticator of the local control, comparative analyses on short-term effects and long-term survivals were performed in patients grouped by the T stage (T3-T4a vs T4b). The short-term effects between the 2 groups were compared using the chi-square test. Survivals were compared with the log-rank test. A two-sided $P$-value of $<0.05$ was considered statistically significant. All statistical analyses were done using IBM SPSS Statistics 23.0 (IBM Co., Armonk, NY, USA).

\section{Results}

\section{Baseline characteristics}

Until March 31, 2017, a total of 60 patients with unresectable locally advanced CC were treated with NACRT before surgery. The pretreatment pathoclinical characteristics of the patients are shown in Table 1. The median age at diagnosis was 56 (range, 29-74) years. The most common site of tumor in this study was also sigmoid (71.7\%). The most common pathologic type was moderately differentiated adenomatous carcinoma (51.7\%). The most common $\mathrm{T}$ stage and $\mathrm{N}$ stage were T4ab (61.7\%) and N2 (61.6\%), respectively. Among the 60 patients, only 1 patient $(1.6 \%)$ had liver metastasis at initial diagnosis. The top 3 common reasons for unresectability were involvement of bladder $(45.0 \%)$, great vessels (18.3\%) and small intestine (15.0\%).

\section{Treatment effects and toxicities}

The short-term treatment effects and toxicities are shown in Table 2. After NACRT, $57(95.0 \%)$ out of the 60 patients

Table I Baseline pathoclinical characteristics of the 60 patients with unresectable colon cancer

\begin{tabular}{ll}
\hline Characteristic & $\mathbf{n}$ \\
\hline Age at diagnosis (range), years & $56(29-74)$ \\
No of patients by sex & \\
Male & $42(70.0 \%)$ \\
Female & $18(30.0 \%)$ \\
No of patients by KPS & \\
90 & $53(88.3 \%)$ \\
80 & $7(11.7 \%)$ \\
No of patients by tumor site & \\
Sigmoid colon & $43(71.7 \%)$ \\
Descending colon & $1(1.6 \%)$ \\
Transverse colon & $4(6.7 \%)$ \\
Ascending colon & $10(16.7 \%)$ \\
Ileocecus & $2(3.3 \%)$ \\
No of patients by differentiation & \\
High & $19(31.7 \%)$ \\
Moderate & $31(51.7 \%)$ \\
Low & $10(16.6 \%)$ \\
No of patients by T stage & \\
T3 & $9(15.0 \%)$ \\
T4a & $14(23.3 \%)$ \\
T4b & $37(61.7 \%)$ \\
No of patients by N stage & \\
No & $1(1.7 \%)$ \\
NI & $22(36.7 \%)$ \\
N2 & $37(61.6 \%)$ \\
No of patients by involved organ & \\
Bladder & $27(45.0 \%)$ \\
Great vessel & $11(18.3 \%)$ \\
Small intestine & $9(15.0 \%)$ \\
Pelvic wall & $4(6.7 \%)$ \\
Liver* & $3(5.0 \%)$ \\
Uterus & $3(5.0 \%)$ \\
Iliopsoas & $2(3.3 \%)$ \\
\hline & $1(1.7 \%)$ \\
\hline 0
\end{tabular}

Note: *Tumor directly invaded into the liver. Abbreviation: KPS, Karnofsky performance score. 
Table 2 Treatment effect and toxicities of neoadjuvant chemoradiotherapy in the 60 patients with unresectable colon cancer

\begin{tabular}{ll}
\hline Characteristic & $\mathbf{n}$ \\
\hline No of patients by surgery type & $35(58.3 \%)$ \\
Simple colectomy & $22(36.7 \%)$ \\
MVR & $3(5.0 \%)$ \\
$\quad$ Surgery abandoned & \\
No of patients by resection grade* & $49(86.0 \%)$ \\
R0 & $3(5.3 \%)$ \\
RI & $5(8.7 \%)$ \\
R2 & \\
No of patients by pCR* & $15(26.3 \%)$ \\
Yes & $42(73.7 \%)$ \\
No & \\
No of patients by down T stage* & $47(82.5 \%)$ \\
Yes & $10(17.5 \%)$ \\
No & \\
No of patients by down N stage* & $53(93.0 \%)$ \\
Yes & $4(7.0 \%)$ \\
No & \\
No of patients by myelosuppression & $48(80.0 \%)$ \\
Grade 0-2 & $12(20.0 \%)$ \\
Grade 3-4 & \\
No of patients by mucositis/dermatitis & $58(96.7 \%)$ \\
Grade 0-2 & $2(3.3 \%)$ \\
Grade 3-4 & \\
No of patients by Gl toxicities & $57(95.0 \%)$ \\
Grade 0-2 & $3(5.0 \%)$ \\
Grade 3-4 of patients by postsurgical complications* & \\
Grade 0-2 & $53(93.0 \%)$ \\
Grade 3-4 & $4(7.0 \%)$ \\
\hline
\end{tabular}

Note: *The proportion was calculated by dividing the number of the patients treated with surgery $(\mathrm{N}=57)$ by the number of the patients in a defined subgroup. Abbreviations: MVR, multivisceral resection; $\mathrm{PCR}$, pathologic complete response; $\mathrm{Gl}$, gastrointestinal.

underwent surgery, and 3 patients (5.0\%) gave up the surgery. Among the patients undergoing surgery, 49 cases (86.0\%) attained an R0 resection. Postsurgical pathology indicated that 15 cases $(26.3 \%)$ achieved pCR, 47 cases $(82.5 \%)$ achieved a down $\mathrm{T}$ stage and 53 cases $(93.0 \%)$ achieved a down N stage (Figure 1).

According to the CTCAE criteria ver. 4.03, the most common grade $3 / 4$ acute toxicities of NACRT were myelosuppression, gastrointestinal (GI) toxicities and mucositis/ dermatitis. No grade 5 acute toxicity was seen. The incidences were $20.0 \%, 5.0 \%$ and $3.3 \%$, respectively. Among the 57 patients receiving surgery, the grade $3 / 4$ Clavien-Dindo postsurgical complications were seen in 4 cases (7.0\%). No grade 5 postsurgical complication was seen, either.

\section{Survival analysis}

The median follow-up time of the patients was 26 (8-80) months. Among the 60 patients, 2 (3.3\%) patients were lost

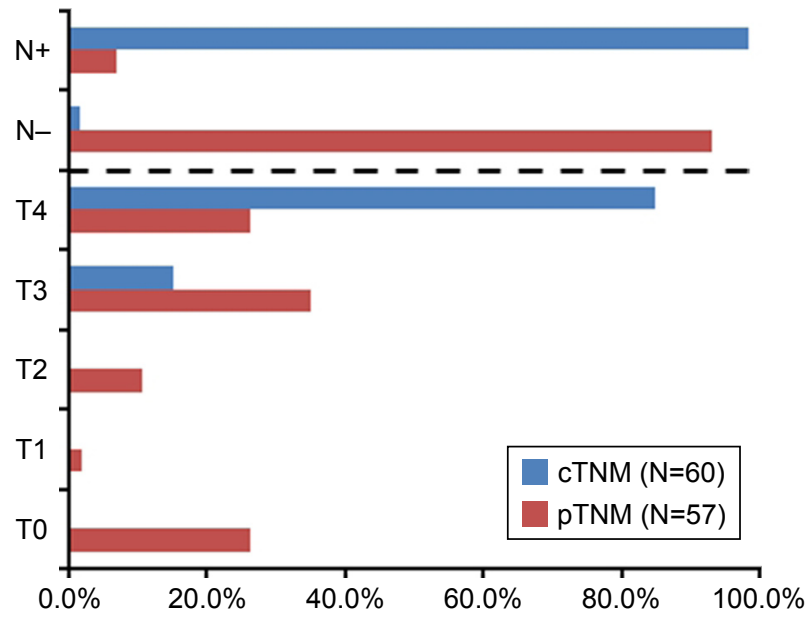

Figure I Stage transfer of the 60 patients with unresectable colon cancer. Notes: Finally, 57 out of the 60 patients received surgery after NACRT. The pretreatment cTNM stage of the 60 patients treated with NACRT and postsurgical PTNM stage of the 57 patients receiving surgery were evaluated.

Abbreviations: cTNM, clinical TNM; NACRT, neoadjuvant chemoradiotherapy; PTNM, pathologic TNM.

to follow-up. Until July 31,2017 , the OS, the DFS, the RFS and the MFS of the patients were $76.7 \%, 68.3 \%, 88.3 \%$ and $70.0 \%$, respectively. The survival curves are shown in Figure 2.

There were totally 7 local recurrences. Among the 49 patients with R0 resection, 5 (10.2\%) cases underwent recurrence. To the contrary, 2 cases $(66.7 \%)$ in the 3 patients with $\mathrm{R} 1$ resection underwent recurrence.

There were totally 18 distant metastases. The most common metastatic site was the liver. Among the 18 metastatic patients, 9 cases $(50.0 \%)$ were finally diagnosed with liver metastasis. There were 5 cases (27.8\%) with lung metastasis, 2 cases $(11.1 \%)$ with metastasis in adrenal gland, 2 cases (11.1\%) with inguinal lymph node metastasis and 2 cases (11.1\%) with bone metastasis. All the 14 deaths in this study were caused by the distant metastasis.

Subgroup analyses in patients with different $\mathrm{T}$ stages showed that pCR rate was higher in the cases with T3-T4a disease than in those with $\mathrm{T} 4 \mathrm{~b}$ disease $(40.9 \%$ vs $14.3 \%$, $P=0.023)$. But no difference was seen in the rate of $\mathrm{R} 0$ resection, down $\mathrm{T}$ or downstage between the 2 groups of patients (Table S1). There was no difference in the OS, the DFS, the RFS or the MFS (Figure S1).

\section{Discussion}

To our knowledge, $32.0 \%-33.3 \%$ of the patients with locally advanced CC have to undergo an MVR, which is considered to improve local control and OS., ${ }^{47}$ Nevertheless, such an intensive treatment only led to a 5 -year OS of $35.1 \% .{ }^{17}$ Not only that, among those managed with MVR, nearly $13.0 \%$ of 
A
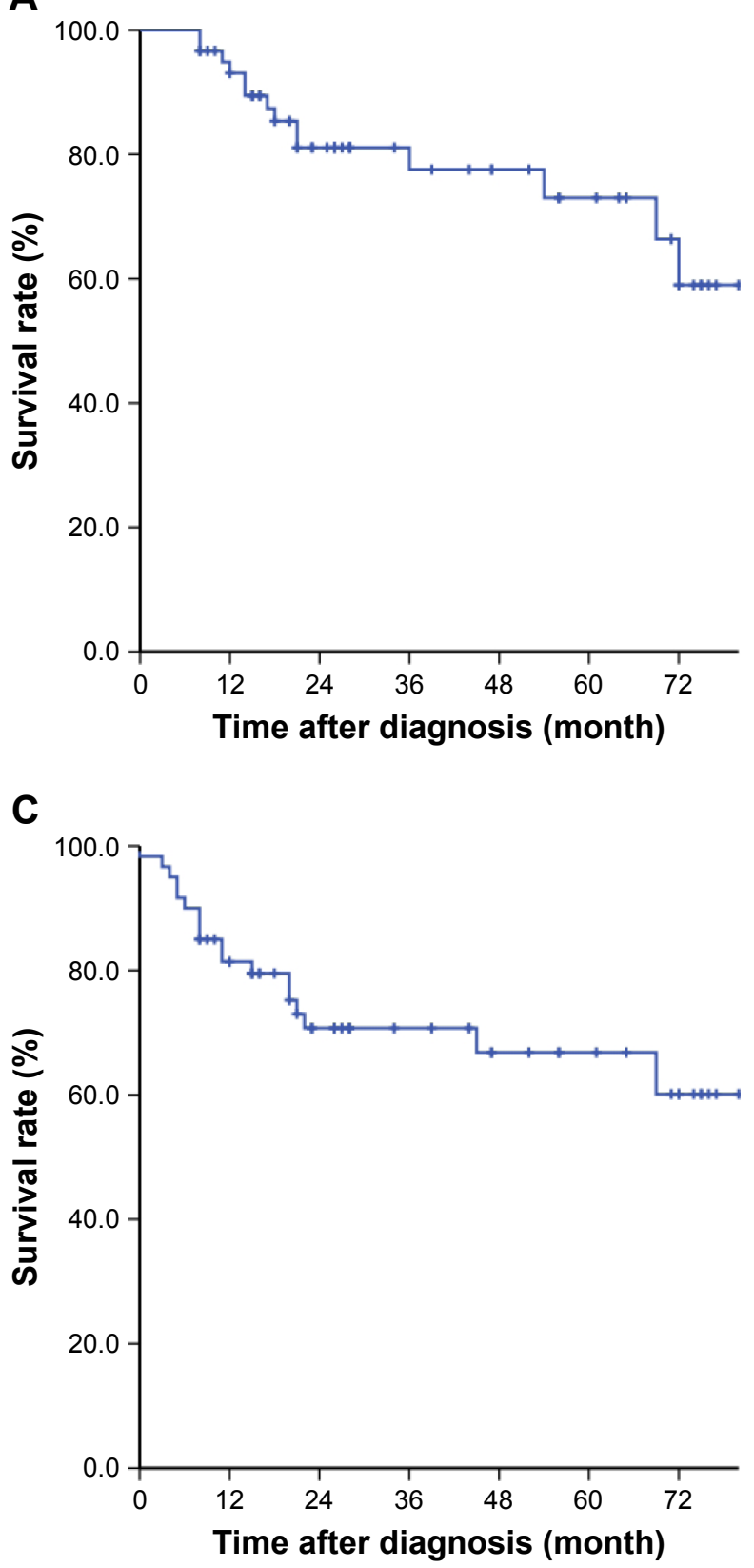

B

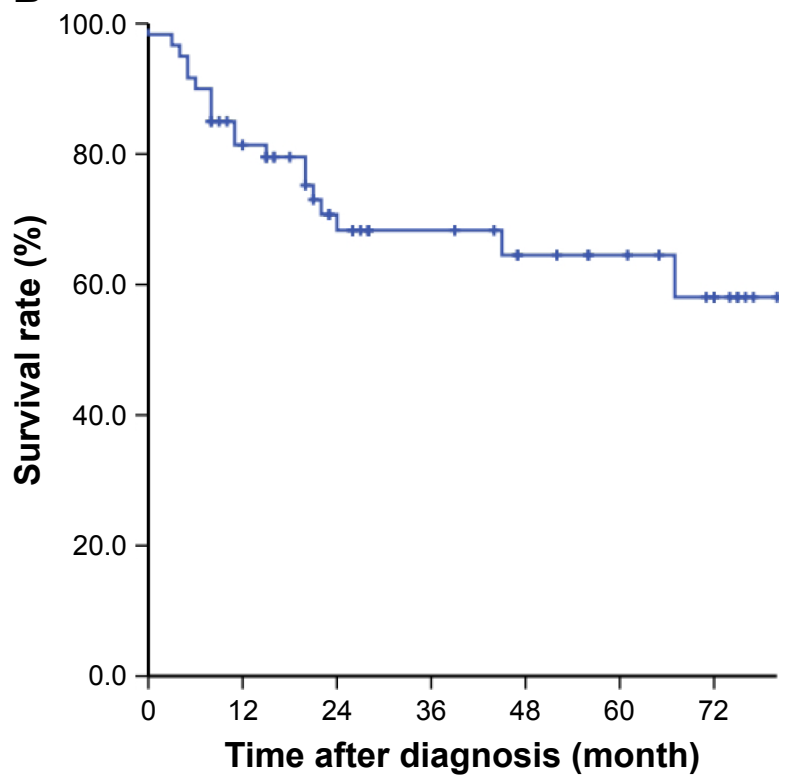

D

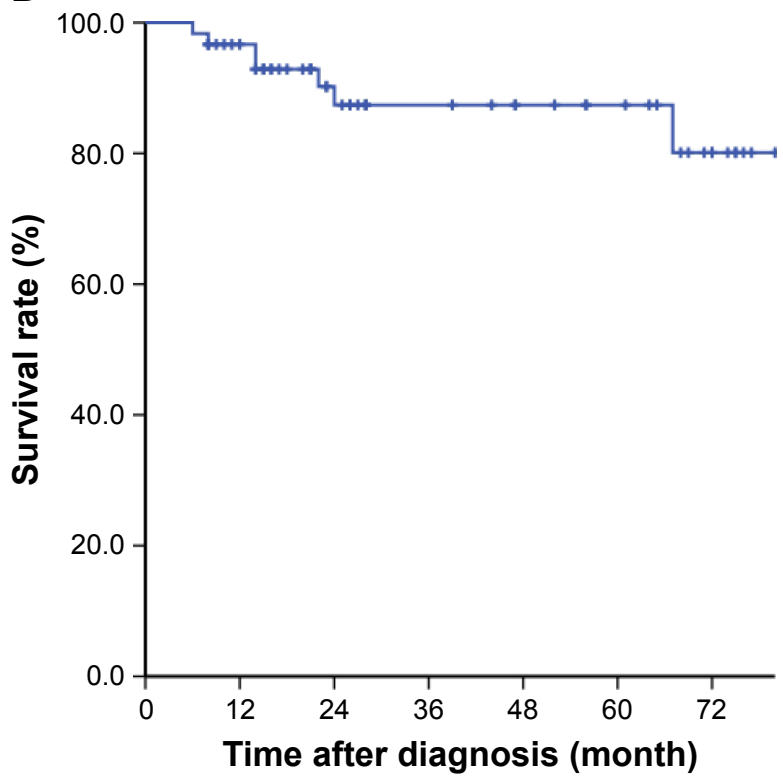

Figure 2 Survival curves of the 60 patients with unresectable colon cancer.

Notes: (A) Overall survival; (B) disease-free survival; (C) distant-metastasis-free survival; (D) local-recurrence-free survival.

the cases failed to achieve an $\mathrm{R} 0$ resection. ${ }^{4}$ There was also a considerable number of patients with unresectable disease. It is known that radicality is a very important prognosticator in $\mathrm{CC} .{ }^{3,18}$ So, the prognosis of these patients was very poor. NACRT might provide patients with locally advanced CC a choice to improve resectability and survival, particularly those with unresectable disease. Our previous works revealed that through NACRT, all the 21 CC patients (100\%) with locally unresectable $\mathrm{CC}$ attained resectability. Of those, only 7 patients $(22.2 \%)$ were in need of a MVR. The rest 14 patients $(66.7 \%)$ received only a simple colectomy, in which the nearby organs could be preserved. The R0 resection was achieved in 20 patients $(95.2 \%)$. The rates of MVR and $\mathrm{R} 0$ resection were close to those with resectable disease. The postsurgical pathology showed that $\mathrm{pCR}$ was observed in 8 patients (38.1\%). After a median follow-up of 42 months, the patients presented with an OS of $95.2 \%{ }^{15}$ Ideal treatment results were also seen in this study. Except 3 patients who abandoned to receive surgery, 57 out of the 60 patients who had locally unresectable CC $(95.0 \%)$ were made resectable after NACRT. Among these 57 patients undergoing surgery, 49 cases (86.0\%) attained an R0 resection. Based on 
postsurgical pathology, the $\mathrm{T}$ stage was down in 47 patients (82.5\%), including 15 cases (26.0\%) exhibiting a pCR.

Distant metastasis is known as the major cause of treatment failure in CC patients. ${ }^{18}$ In this study, all the 14 deaths were due to distant metastasis. The regional lymph node metastasis is one of the most important risk factors of distant metastasis. ${ }^{19}$ NACRT was proved in this study not only to improve the resectability but also to eliminate the lymph node metastasis. The $\mathrm{N}$ stage was down in 53 cases (93.0\%). Therefore, NACRT might help to reduce the distant metastasis as well. In fact, when followed up for a median period of 26 months, the patients in this study presented with OS, DFS, RFS and MFS of $76.7 \%, 68.3 \%, 88.3 \%$ and $70.0 \%$, respectively. The 2 -year OS and DFS of stage III $\mathrm{CC}$ are reported as $65.9 \%$ and $59.2 \%$, respectively, in the literature. ${ }^{20}$ The figures of the resectable tumors are $94.1 \%$ and $77.5 \%{ }^{21}$ Our results of follow-up indicated that NACRT made the survivals of the patients with unresectable disease improve towards those of the patients with resectable disease. In other words, the short-term treatment effects of NACRT did translate into a relatively long-term survival benefit. Additionally, the treatment results might also be influenced by the pretreatment $\mathrm{T}$ stage. NACRT led to a higher $\mathrm{pCR}$ rate in patients with earlier T (T3-T4a) stage. But the resection radicality, downstage rate and long-term survivals seemed to have no difference between patients with different $\mathrm{T}$ stages. In this study, even those with unresectable $\mathrm{T} 4 \mathrm{~b}$ disease appeared to have an $\mathrm{R} 0$ resection rate $(77.1 \%)$ approximate to the resectable disease, and OS (74.3\%) and DFS (65.7\%) better than the average level of the stage III CC.

Indeed, the adverse reactions will be another concern when NACRT is applied. As we know, IMRT has the abilities of delivering irradiation precisely to the tumors, and decreasing the dose simultaneously to the adjacent important organs, such as the small intestine, liver and kidneys. The satisfactory safety of IMRT has already been shown in treating locally advanced $\mathrm{RC},{ }^{22}$ In our previous works, only 1 of the 21 unresectable $C C$ patient (4.8\%) had grade 3 hand-foot syndrome. No grade 4 acute toxicity due to NACRT was seen, neither was grade $3 / 4$ postsurgical complication. ${ }^{15}$ In this study, the most common grade $3 / 4$ acute toxicities included myelosuppression (20.0\%), GI reaction $(5.0 \%)$, and mucositis/dermatitis (3.3\%). The incidence of grade $3 / 4$ postsurgical complication was $7.0 \%$. No grade 5 adverse event was observed. Considering that $6.9 \%-18.8 \%$ of the locally advanced $\mathrm{CC}$ patients had postsurgical complications in need of re-operation, ${ }^{4,5}$ NACRT appeared as a tolerable treatment modality for locally advanced CC.
Actually, NACRT did not only improve the prognosis of unresectable $\mathrm{CC}$ but also ameliorate the organ preservation during surgery. The small intestine and the bladder are reported as the top 2 frequently affected organs in locally advanced CC (incidence rates are as high as $48 \%$ and $50 \%$, respectively). ${ }^{23}$ They are also the organs most commonly removed in the MVR (resection rates are $31.6 \%$ and $27.0 \%$, respectively). ${ }^{5}$ In our previous study, the bladder appeared as the most commonly involved organ. There were 10 patients (47.6\%) suffering from bladder invasion. Among the patients receiving surgery, only 6 cases $(28.5 \%)$ received only a partial cystectomy. No one received a total cystectomy. Cystectomy was avoided in 4 patients, owing to NACRT. ${ }^{15}$ In this study, bladder was involved in 27 patients $(45.0 \%)$ and small intestine in 9 patients $(15.0 \%)$. Of the 57 patients who underwent surgery, 22 cases $(38.6 \%)$ received a partial cystectomy. No patient received a total cystectomy, either. Partial resection of small intestine was performed in 5 patients (8.8\%). Only 1 patient (1.8\%) received Whipple's resection. The resection rates of the bladder and small intestine were similar to the historical data of the resectable disease. In a word, patients with locally unresectable CC might achieve benefit of postoperative life quality from NACRT.

Prior to our studies, approaches have been made to improve prognosis of locally advanced CC. Although there have been evidences supporting the application of neoadjuvant chemotherapy, ${ }^{24-26}$ the clinical value of NACRT remained uncertain. Moreover, there were few researches focusing on NACRT in unresectable CC. They were almost case reports and retrospective studies with a small size of sample. In the literature, the earliest case report in this field dated back to the year of 2007. Mizukami et al administered NACRT to a patient with unresectable sigmoid CC and made him suitable to receive a low anterior resection. ${ }^{27}$ Encouraging treatment effects were also seen in the case reports by Yoh et al and Yoshitomi et al. ${ }^{28,29}$ Burton et al retrospectively reviewed 75 patients with locally advanced sigmoid CC, including 18 cases with unresectable disease. After NACRT, $50 \%$ of the unresectable tumors became resectable. The 3 -year OS of the patients treated with NACRT was close to that of the patients with resectable disease. ${ }^{30}$ The study of Cukier et al revealed in 33 locally advanced CC (22 unresectable) that NACRT resulted in an $\mathrm{R} 0$ resection rate of $100 \%$ and a good local control, together with acceptable morbidity and mortality. ${ }^{31}$ Huang et al also showed in 34 patients (2 unresectable) that NACRT brought a prominent $\mathrm{pCR}$ rate with acceptable toxicities. ${ }^{32}$ We proved that NACRT was effective and safe even in locally unresectable CC, through 
a relative large scale of prospective collected data. It is the strength of our study. The results might provide evidences for oncological physicians to direct treatment strategies and conduct clinical trials for locally advanced CC. However, there are still 2 main limitations in this study. First, it was not a controlled study with random allocation of the patients. Second, the follow-up time was relatively short. Thus, a randomized controlled trial with a long follow-up period is needed to further validate the results of this study, before popularization to clinical use.

\section{Conclusion}

For patients with locally unresectable CC, NACRT might bring a high $\mathrm{R} 0$ resection, downstage and $\mathrm{pCR}$ rate, which might translate into an ideal long-term outcome and satisfactory organ preservation. Meanwhile, the NACRT-related toxicities and surgery-related morbidity are acceptable.

\section{Disclosure}

The authors report no conflicts of interest in this work.

\section{References}

1. Chen W, Zheng R, Baade PD, et al. Cancer statistics in China, 2015. CA Cancer J Clin. 2016;66(2):115-132.

2. NCCN Clinical Practice Guidelines in Oncology (NCCN Guidelines $\left.{ }^{\circledR}\right)$ : Colon Cancer (version 2. 2017). NCCN.org. Available from: https://www. nccn.org/store/login/login.aspx?ReturnURL=https:/www.nccn.org/ professionals/physician_gls/pdf/colon.pdf. Accessed March 31, 2017.

3. Tie J, Wang Y, Tomasetti C, et al. Circulating tumor DNA analysis detects minimal residual disease and predicts recurrence in patients with stage II colon cancer. Sci Transl Med. 2016;8(346):346ra92.

4. Klaver CE, Gietelink L, Bemelman WA, et al; Dutch Surgical Colorectal Audit Group. Locally advanced colon cancer: evaluation of current clinical practice and treatment outcomes at the population level. $J$ Natl Compr Canc Netw. 2017;15(2):181-190.

5. Croner RS, Merkel S, Papadopoulos T, Schellerer V, Hohenberger W, Goehl J. Multivisceral resection for colon carcinoma. Dis Colon Rectum. 2009;52(8):1381-1386.

6. van der Sijp MP, Bastiaannet E, Mesker WE, et al. Differences between colon and rectal cancer in complications, short-term survival and recurrences. Int J Colorectal Dis. 2016;31(10):1683-1691.

7. Breugom AJ, van Dongen DT, Bastiaannet E, et al. Association between the most frequent complications after surgery for stage I-III colon cancer and short-term survival, long-term survival, and recurrences. Ann Surg Oncol. 2016;23(9):2858-2865.

8. Mathis KL, Nelson H, Pemberton JH, Haddock MG, Gunderson LL. Unresectable colorectal cancer can be cured with multimodality therapy. Ann Surg. 2008;248(4):592-598.

9. DE Felice F, Musio D, Benevento I, et al. Influence of organ invasion in clinical outcomes for locally advanced rectal cancer. Anticancer Res. 2016;36(10):5443-5447.

10. Sineshaw HM, Jemal A, Thomas CR Jr, Mitin T. Changes in treatment patterns for patients with locally advanced rectal cancer in the United States over the past decade: an analysis from the National Cancer Data Base. Cancer. 2016;122(13):1996-2003.

11. Mohammed S, Anaya DA, Awad SS, Albo D, Berger DH, Artinyan A. Sphincter preservation rates after radical resection for rectal cancer in the United States veteran population: opportunity for improvement in early disease. Ann Surg Oncol. 2015;22(1):216-223.
12. NCCN Clinical Practice Guidelines in Oncology $\left(\mathrm{NCCN}\right.$ Guidelines $\left.\mathrm{s}^{\circledR}\right)$ : Rectal Cancer (version 3. 2017). NCCN.org. Available from: https://www. nccn.org/store/login/login.aspx?ReturnURL=https://www.nccn.org/ professionals/physician_gls/pdf/rectal.pdf. Accessed March 31, 2017.

13. Huang C, Shen JC, Zhang J, et al. Clinical comparison of laparoscopy vs open surgery in a radical operation for rectal cancer: a retrospective casecontrol study. World J Gastroenterol. 2015;21(48):13532-13541.

14. Thuraisingam R, Jandova J, Pandit V, Michailidou M, Nfonsam VN. Assessing the national trends in colon cancer among Native Americans: A 12 year SEER database study. Am J Surg. 2017;214(2):228-231.

15. Qiu B, Ding PR, Cai L, et al. Outcomes of preoperative chemoradiotherapy followed by surgery in patients with unresectable locally advanced sigmoid colon cancer. Chin J Cancer. 2016;35(1):65.

16. Sobin LH, Gospodarowicz MK, Wittekind C. TNM Classification of Malignant Tumours. (UICC International Union Against Cancer). 7th ed. New York: Wiley-Blackwell; 2009.

17. Govindarajan A, Coburn NG, Kiss A, Rabeneck L, Smith AJ, Law CH. Population-based assessment of the surgical management of locally advanced colorectal cancer. J Natl Cancer Inst. 2006; 98(20):1474-1481.

18. Andreoni B, Chiappa A, Bertani E, et al. Surgical outcomes for colon and rectal cancer over a decade: results from a consecutive monocentric experience in 902 unselected patients. World J Surg Oncol. 2007; $5: 73$.

19. Cho T, Shiozawa E, Urushibara F, et al. The role of microvessel density, lymph node metastasis, and tumor size as prognostic factors of distant metastasis in colorectal cancer. Oncol Lett. 2017;13(6):4327-4333.

20. Rashtak S, Ruan X, Druliner BR, et al. Peripheral neutrophil to lymphocyte ratio improves prognostication in colon cancer. Clin Colorectal Cancer. 2017;16(2):115-123.

21. Emile JF, Julié C, Le Malicot K, et al; PETACC8 Study Investigators; Austrian Breast and Colorectal cancer Study Group (ABCSG); Belgian Group of Digestive Oncology (BGDO); Lone Nørgård Petersen; Fédération Francophone de Cancérologie Digestive (FFCD); Fédération Nationale des Centres de Lutte Contre le Cancer (UNICANCER); Fédération Nationale des Centres de Lutte Contre le Cancer Association Européenne de Recherche en Oncologie (AERO); Arbeitsgemeinschaft Internistische Onkologie (AIO); Gruppo Italiano per lo Studio dei Carcinomi dell'Apparato Digerente (GISCAD); Gruppo Oncologico dell'Italia Meridionale (GOIM); Istituto Oncologico Romagnolo (IOR); Gruppo Cooperativo Chirurgico Italiano (GOCCI); Gruppo Oncologico Nord Ovest (GONO); Gruppo Oncologico Italiano di Ricerca Clinica (GOIRC); Gruppo Cooperativo do Cancro Digestivo da Associação Portuguesa de Investigação Oncológica (GCCD, APIO); Grupo Español para el Tratamiento de los Tumores Digestivos (TTD); John Allen Bridgewater. Prospective validation of a lymphocyte infiltration prognostic test in stage III colon cancer patients treated with adjuvant FOLFOX. Eur J Cancer. 2017;82:16-24.

22. Stuyck C, Wegge M, Bulens P, Joye I, Haustermans K. Moderate dose escalation with volumetric modulated arc therapy improves outcome in rectal cancer. Acta Oncol. 2017;56(11):1501-1506.

23. Lee-Kong S, Lisle D. Surgical management of complicated colon cancer. Clin Colon Rectal Surg. 2015;28(4):228-233.

24. Foxtrot Collaborative Group. Feasibility of preoperative chemotherapy for locally advanced, operable colon cancer: the pilot phase of a randomised controlled trial. Lancet Oncol. 2012;13(11):1152-1160.

25. Zhou H, Song Y, Jiang J, et al. A pilot phase II study of neoadjuvant triplet chemotherapy regimen in patients with locally advanced resectable colon cancer. Chin J Cancer Res. 2016;28(6):598-605.

26. Jakobsen A, Andersen F, Fischer A, et al. Neoadjuvant chemotherapy in locally advanced colon cancer. A phase II trial. Acta Oncol. 2015;54(10): 1747-1753.

27. Mizukami H, Yoshizawa Y, Sasaya S, Nemoto H, Maezawa K, Sanada Y. [A case of advanced colon cancer invading the rectum effectively treated with chemoradiation therapy before surgery]. Gan To Kagaku Ryoho. 2007;34(6):953-956. Japanese [with English abstract]. 
28. Yoh T, Yamamichi K, Oishi M, Iwaki R, Motohiro T. [A case of effective neoadjuvant chemoradiotherapy with capecitabine for locally advanced sigmoid colon cancer]. Gan To Kagaku Ryoho. 2011;38(6):1021-1024. Japanese [with English abstract].

29. Yoshitomi M, Hashida H, Nomura A, Ueda S, Terajima H, Osaki N. A case of locally advanced sigmoid colon cancer treated with neoadjuvant chemoradiotherapy. Gan To Kagaku Ryoho. 2014;41(9):1175-1178. Japanese [with English abstract].

30. Burton S, Brown G, Daniels I, et al. MRI identified prognostic features of tumors in distal sigmoid, rectosigmoid, and upper rectum: treatment with radiotherapy and chemotherapy. Int J Radiat Oncol Biol Phys. 2006;65(2):445-451.
31. Cukier M, Smith AJ, Milot L, et al. Neoadjuvant chemoradiotherapy and multivisceral resection for primary locally advanced adherent colon cancer: a single institution experience. Eur J Surg Oncol. 2012;38(8):677-682.

32. Huang CM, Huang MY, Ma CJ, et al. Neoadjuvant FOLFOX chemotherapy combined with radiotherapy followed by radical resection in patients with locally advanced colon cancer. Radiat Oncol. 2017;12(1):48. 


\section{Supplementary materials}

The target definition, delineation and dosage standard of radiotherapy in our hospital

All the patients in this study were immobilized by an AIO Bellyboard and Pelvic Solution system (AIO solution, Orfit Industries, Wijnegem, Belgium). Patients were simulated with a moderately full bladder. After a computed tomography (CT) simulation with $3 \mathrm{~mm}$ slice thickness, the target volumes of the intensity-modulated radiotherapy (IMRT) were delineated according to the guidelines of the International Commission on Radiation Units and Measurements Reports 50 and 62. The gross tumor volume (GTV) was the macroscopic tumor and the enlarged lymph nodes visible on magnetic resonance imaging or CT. The clinical target volume (CTV) covered the GTV with a radial margin of $2 \mathrm{~cm}$, and included the corresponding mesocolon and the high-risk regions of lymphatic drainage. If the tumor invaded adjacent structures, a further $1.5 \mathrm{~cm}$ isotropic margin into the involved structures would be included in the CTV to account for the microscopic disease. Planning target volumes (PTVs) for GTV and CTV covered the GTV and CTV with an isotropic margin of $0.6 \mathrm{~cm}$.

Radiotherapy was done in a conventional fractionation (2 Gy per fraction, 1 fraction per day, 5 days per week), in which the total dose of PTVs for GTV and CTV were 50

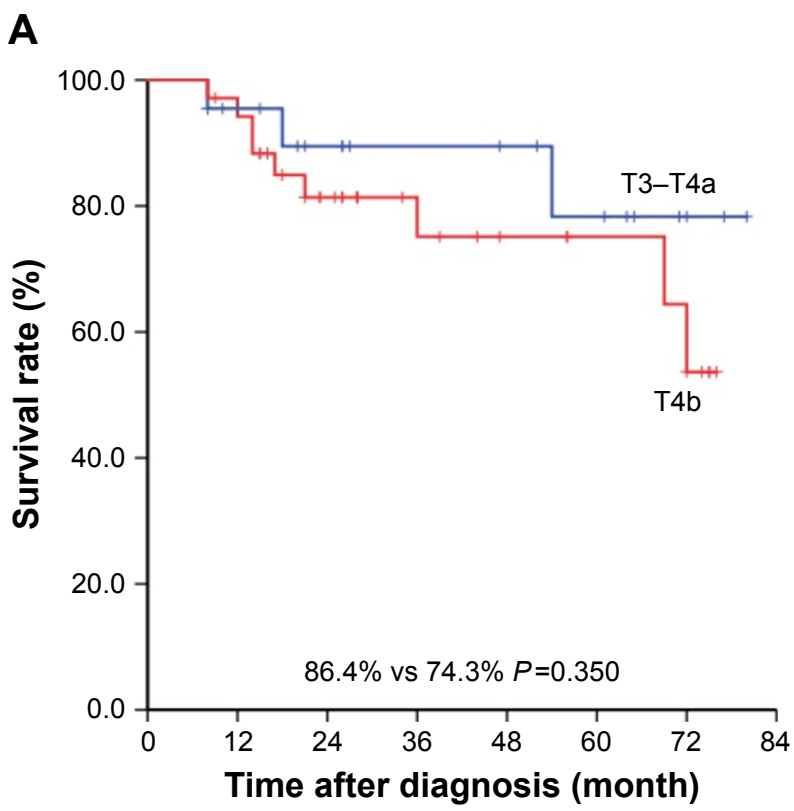

Figure SI (Continued)
Table SI Comparison of short-term effects of NACRT in patients with different $\mathrm{T}$ stages (T3-T4a vs T4b)

\begin{tabular}{lllll}
\hline & T3-T4a (N=22) & T4b (N=35) & $X^{2}$ & $P$-value \\
\hline $\begin{array}{l}\text { R0 resection } \\
\text { Yes }\end{array}$ & $19(86.4 \%)$ & $30(77.1 \%)$ & 0.005 & \\
$\quad$ No & $3(13.6 \%)$ & $5(14.3 \%)$ & & \\
Down T & & & & 0.991 \\
$\quad$ Yes & $17(77.3 \%)$ & $27(77.1 \%)$ & 0.000 & \\
$\quad$ No & $5(22.7 \%)$ & $8(22.9 \%)$ & & \\
Downstage & $17(77.3 \%)$ & $30(85.7 \%)$ & 0.665 & \\
$\quad$ Yes & $5(22.7 \%)$ & $5(14.3 \%)$ & & \\
$\quad$ No & & & & $0.023 *$ \\
PCR & $9(40.9 \%)$ & $5(14.3 \%)$ & 5.168 & \\
Yes & $13(59.1 \%)$ & $30(85.7 \%)$ & & \\
No & & & & \\
\hline
\end{tabular}

Note: $* p<0.05$.

Abbreviations: NACRT, neoadjuvant chemoradiotherapy; $\mathrm{PCR}$, pathologic complete response.

and 46 Gy, respectively. A linear accelerator delivering an $8 \mathrm{MV}$ photon beam was used to perform the IMRT. Dose constraints of the organ at risk included the following: 1) maximal dose (Dmax) was less than 52 Gy, and volume receiving dose $>50$ Gy (V50) was less than $5 \%$ for the small intestine; 2) V50 of the bladder was less than 50\%; 3) volume receiving dose $>20$ Gy (V20) was less than $30 \%$ for each kidney; 4) volume receiving dose $>30 \mathrm{~Gy}$ (V30) of the liver was less than 60\%; and 5) V50 was less than 5\% for femoral head of each side.

B

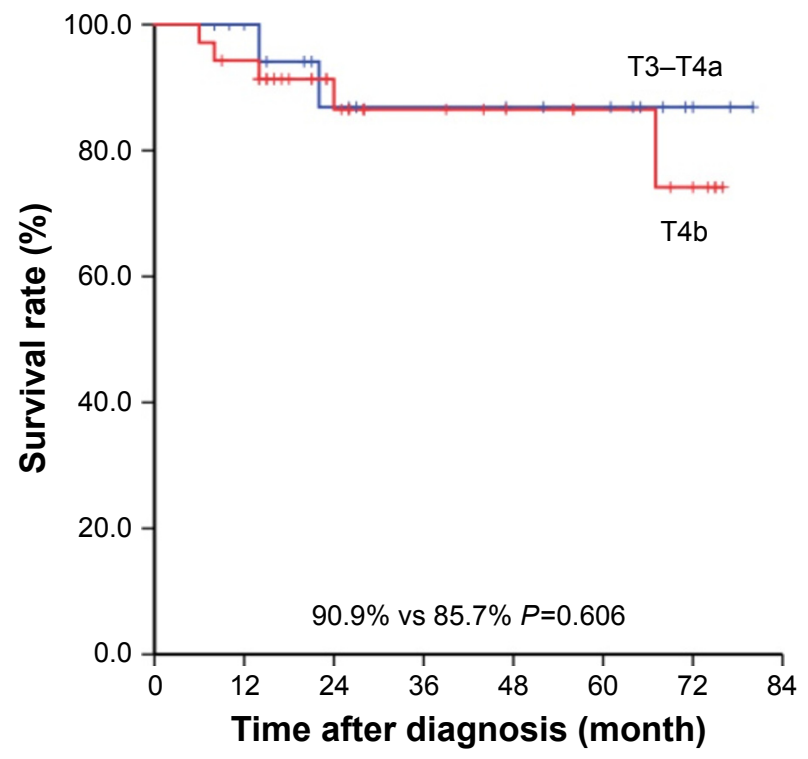



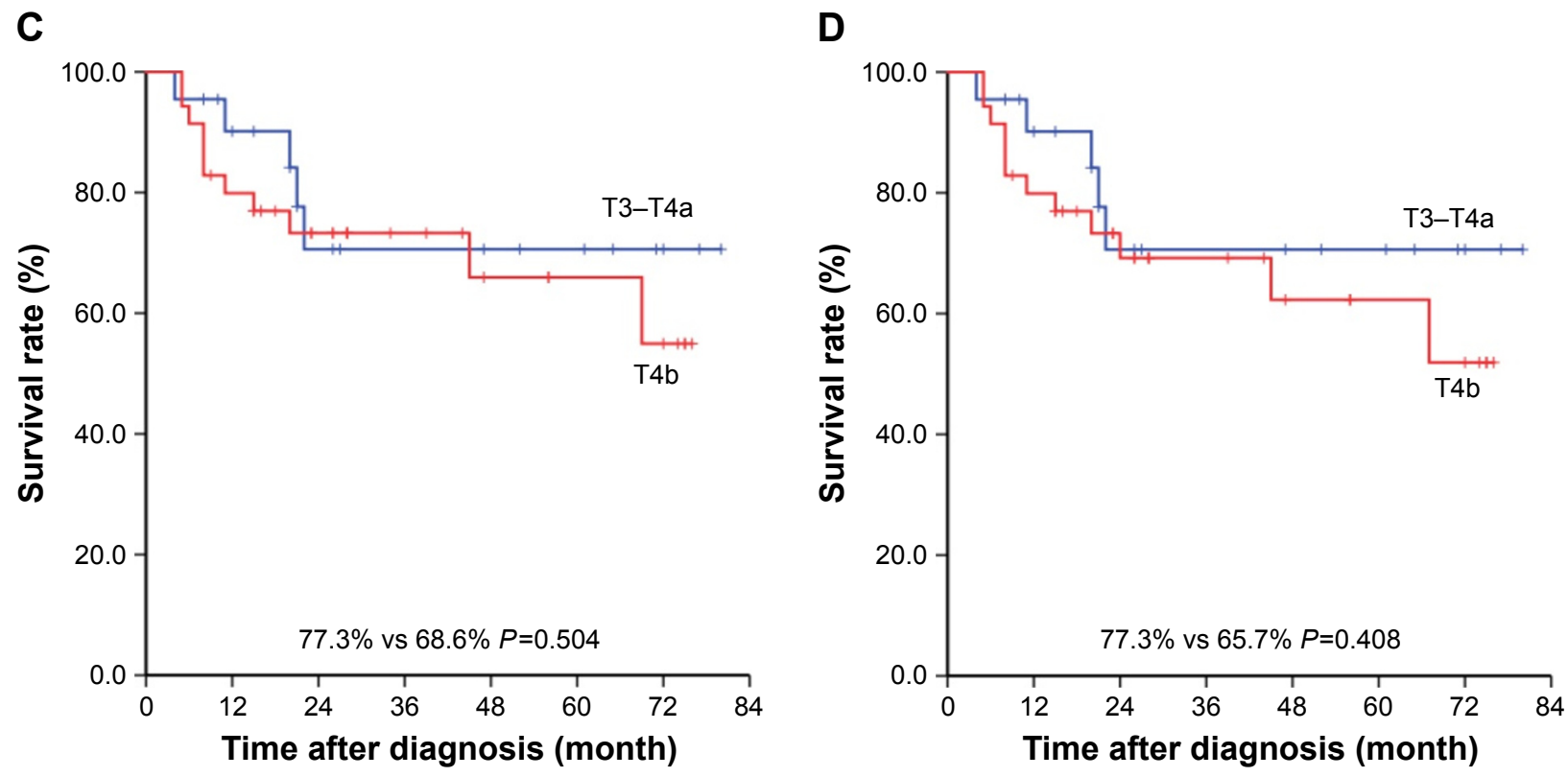

Figure SI Comparison in long-term survivals of NACRT in patients with different T stages (T3-T4a vs T4b).

Note: There was no difference in the overall (A), the local-recurrence-free (B), the distant-metastasis-free (C) and the disease-free (D) survivals.

Abbreviation: NACRT, neoadjuvant chemoradiotherapy.

\section{Publish your work in this journal}

OncoTargets and Therapy is an international, peer-reviewed, open access journal focusing on the pathological basis of all cancers, potential targets for therapy and treatment protocols employed to improve the management of cancer patients. The journal also focuses on the impact of management programs and new therapeutic agents and protocols on

patient perspectives such as quality of life, adherence and satisfaction. The manuscript management system is completely online and includes a very quick and fair peer-review system, which is all easy to use. Visit http://www.dovepress.com/testimonials.php to read real quotes from published authors. 